\title{
EXIT-Chart Aided Near-Capacity Irregular Bit-Interleaved Coded Modulation Design
}

\author{
Ronald Y. S. Tee, Robert G. Maunder, and Lajos Hanzo
}

\begin{abstract}
A near-capacity irregular Bit-Interleaved Coded Modulation based Iterative Decoding (Ir-BICM-ID) aided scheme is proposed. The irregular design of the scheme pervades the three basic components of BICM-ID, namely the encoder, the unity-rate precoder and the bit-to-symbol mapper. As a result, irregular BICM-ID schemes constituted by irregular components are created, which are capable of approaching the capacity of coded modulation. This is achieved by creating a marginally open EXtrinsic Information Transfer (EXIT) chart tunnel, and exploiting the theorem that the open tunnel's area is characteristic of how closely the scheme operates to the channel's capacity. The proposed Ir-BICM-ID scheme employs Irregular Convolutional Codes (IrCC), Irregular Unity-Rate Codes (IrURC) and Irregular Mappers (IrMapper).
\end{abstract}

Index Terms-EXIT-chart, near-capacity system performance, irregular codes, bit-interleaved coded modulation, BICM.

\section{INTRODUCTION}

B IT-INTERLEAVED Coded Modulation using Iterative Decoding (BICM-ID) [1] was originally designed as a bandwidth efficient coded modulation scheme for Rayleigh fading channels. It consists of three main blocks - a convolutional encoder, bit-interleavers and a bit-to-symbol mapper, as detailed in [2], [3]. Iterative detection was achieved by exchanging soft extrinsic information between the symbol-tobit demapper and the decoder. The Bit Error Ratio (BER) versus $E_{b} / N_{0}$ curve of BICM-ID scheme with the achievable convergence performance can be characterised with the aid of EXtrinsic Information Transfer (EXIT) charts [4].

Different bit-to-symbol mapping schemes have been investigated in order to improve the convergence behaviour of BICMID [5] by shaping the demapper's EXIT characteristic for the sake of creating an open EXIT tunnel and hence to achieve an infinitesimally low BER. Furthermore, an adaptive BICM arrangement using various iterative decoding schemes was proposed in [6], which employed different signal constellations and bit-to-symbol mapping schemes within one codeword. This flexible signalling scheme required the knowledge of the average signal quality at the transmitter, for invoking advanced Adaptive Modulation and Coding (AMC) [6] in order to improve the overall BICM-ID scheme's achievable performance.

A Unity-Rate Code (URC) can be used as a precoder for creating an Infinite Impulse Response (IIR) inner demapper component in order to reach the $(1,1)$ EXIT chart convergence

Manuscript received October 28, 2007; revised March 5, 2008; accepted May 1, 2008. The associate editor coordinating the review of this letter and approving it for publication was M. Torlak.

The authors are with the School of ECS, University of Southampton, SO17 1BJ, UK (e-mail: 1h@ecs.soton.ac.uk).

Digital Object Identifier 10.1109/T-WC.2009.071202 point and hence to achieve an infinitesimally low BER. A precoded mapper scheme constituted by the rate-1 precoder [6] was also proposed for a three-stage design constituted by an encoder, precoder and modulator.

Against this background, the novel contribution of this Letter is that we propose an irregular BICM-ID arrangement for the sake of achieving a near-capacity performance. Our approach is based on invoking EXIT chart analysis for minimising the area of the open EXIT tunnel. The classic outer convolutional encoder is replaced by an Irregular Convolutional Code (IrCC) [7] [8]. Furthermore, low-complexity Convolutional Codes (CC) together with a hybrid combination of memoryless repetition codes are invoked for creating the required EXIT curve shapes. In the same spirit, the inner component code consists of an Irregular Unity Rate Code (IrURC) combined with an Irregular Mapper (IrMapper). The inner iterations are invoked between the IrURC having a code rate of unity as well as up to three shift-register stages and the IrMapper employing different mapping schemes for the sake of creating the required diverse range of EXIT curve shapes. A high-flexibility inner-outer EXIT chart matching algorithm is used for creating a marginally open EXIT tunnel.

The rest of this contribution is organised as follows. Section II provides an overview of our proposed Ir-BICM-ID scheme. Our EXIT chart analysis is presented in Section III and the irregular components of the Ir-BICM-ID scheme are discussed in Section IV. The EXIT matching algorithm advocated is described in Section V. In Section VI, we quantify the achievable performance of this novel scheme, invoking the IrCC, IrURC and IrMapper, while our conclusions are presented in Section VII.

\section{System Overview}

The schematic of the proposed Ir-BICM-ID scheme is shown in Figure 1, which is constituted by the three main component blocks, namely the IrCC, the bit interleaver and the mapper. In our Ir-BICM-ID design, the IrCC is constituted by $P$ number of low-complexity convolutional constituent codes $(C C)$. The inner component module of Figure 1 contains $Q$ number of URCs and $Q$ number of mappers $(M)$. This irregular mapping scheme is referred to as the IrMapper.

At the receiver, an iterative decoder is invoked, exchanging extrinsic information between the inner and outer components. The inner iterations exchange extrinsic information between the irregular demapper and the IrURC decoder, where the notation $A($.$) represents the a priori information$ quantified in terms of Log-Likelihood Ratios (LLRs), while $E($.$) denotes the extrinsic information also expressed in$ 


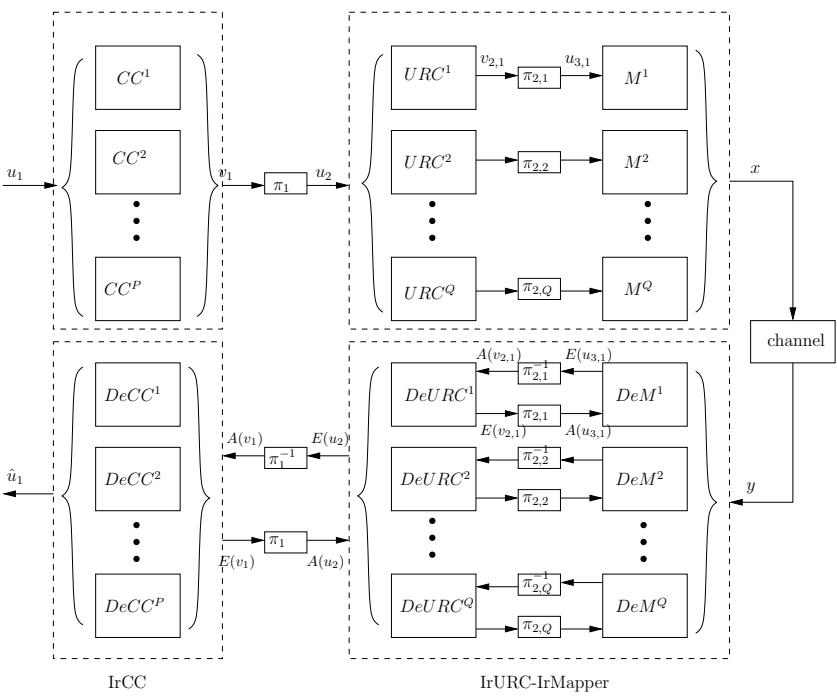

Fig. 1. The Ir-BICM-ID scheme. The binary source bit stream $u_{1}$ is encoded by the $\mathrm{IrCC}$ encoder and the encoded bits $v_{1}$ are interleaved by the bit interleaver $\pi_{1}$, yielding the permuted bits $u_{2}$. Each of the subcomponent $U R C^{i}$ code yields the encoded bits $v_{2, i}$ according to the assigned weighting coefficients, where $i$ denotes the component index. The resultant bits $v_{2, i}$ are interleaved by the corresponding bit interleaver $\pi_{2, i}$ and the permuted bits $u_{3, i}$ are then mapped to the input of the associated mapper $M^{i}$. The modulated symbols $x$ are transmitted via a Rayleigh fading channel and the received signals $y$ are demodulated.

terms of LLRs. Observe in Figure 1 that each component of the URC decoder and demapper is termed as DeURC $C^{i}$ and $D e M^{i}$, respectively and only the first component is labeled to avoid obfuscation. The inner component is represented by the IrURC-IrMapper block.

By contrast, the outer iterations are invoked between the IrURC decoder and the IrCC decoder of Figure 1. Here, each of the IrCC component decoder is termed as $D e C C^{i}$. Since both the IrURC and IrCC schemes are trellis-based, their decoders employ the Maximum A-Posteriori (MAP) algorithm. The inner iterative decoder continues iterating, until no more mutual information improvement is achieved. This is then followed by outer iterative decoding, which would generate new a priori information for the next inner iteration. This process continues until the required number of total iteration is reached.

\section{EXIT CHART ANALYSIS}

EXIT chart analysis is performed to characterise the achievable iterative decoding convergence performance. The IrBICM-ID scheme is a three-stage arrangement, which requires 3-Dimensional (3D) EXIT analysis, as presented in [9]. However, since the IrURC decoder and the Irregular Demapper (IrDemapper) constitute low-complexity components, the lowcomplexity inner iterations are continued until the mutual information $E\left(v_{2, i}\right)$ becomes near-constant, because no more extrinsic information may be gleaned by any of these two components without the third component's intervention. Hence, we can simplify the three-stage EXIT chart representation to a 2Dimensional (2D) EXIT curve, as detailed below.

Let $I_{A(b)}$ represent the mutual information between the a priori information $A(b)$ and the bit value $b$, while $I_{E(b)}$ denotes the mutual information between the extrinsic information $E(b)$ and the bit $b$. Observe from Figure 1 that the EXIT function of the Ir-Demapper can be represented as a function of $I_{E\left(u_{3}\right)}$ and the channel's $E_{b} / N_{0}$ value [4] as $I_{E\left(u_{3}\right)}=\frac{1}{Q} \sum_{i=1}^{Q} f_{u_{3}}\left[I_{A\left(u_{3, i}\right)}, E_{b} / N_{0}\right]$.

Observe in Figure 1 that in a typical three-stage concatenated design, the IrURC decoder has two mutual information outputs, namely $I_{E\left(u_{2}\right)}$ and $I_{E\left(v_{2}\right)}$, where $I_{E\left(v_{2}\right)}$ is the total mutual information of $I_{E\left(v_{2, i}\right)}, \forall i$. Both of these mutual information components depend on two a priori mutual information inputs, namely on $I_{A\left(u_{2}\right)}$ and $I_{A\left(v_{2}\right)}$. The two functions can be defined as $I_{E\left(u_{2}\right)}=f_{u_{2}}\left[I_{A\left(u_{2}\right)}, I_{A\left(v_{2}\right)}\right]$ and $I_{E\left(v_{2}\right)}=f_{v_{2}}\left[I_{A\left(u_{2}\right)}, I_{A\left(v_{2}\right)}\right]$.

In this Ir-BICM-ID scheme, we continue invoking inner iterations, until we succeed in generating sufficiently reliable mutual information $I_{E\left(u_{2}\right)}$, before activating the outer iterations. Hence we may combine the inner component blocks according to Figure 1, where the dotted box indicates the inner IrURC-IrMapper component. The EXIT function of this inner component can be defined by $I_{E\left(u_{2}\right)}=f_{u_{2}}\left[I_{A\left(u_{2}\right)}, E_{b} / N_{0}\right]$. For the IrCC decoder, the EXIT function becomes $I_{E\left(v_{1}\right)}=$ $f_{v_{1}}\left[I_{A\left(v_{1}\right)}\right]$.

The so-called area-property of EXIT charts [10] can be exploited for creating a near-capacity Ir-BICM-ID scheme based on EXIT curve matching. The area property of EXIT charts [10] states that the area under the EXIT curve of an inner decoder component is approximately equal to the attainable channel capacity, provided that the channel's input symbols are equiprobable. As for the outer component, the area under its EXIT function is equivalent to $\left(1-R_{o}\right)$, where $R_{O}$ is the outer component's coding rate. The area properties were formally shown to hold for the Binary Erasure Channel (BEC), but there is experimental evidence that it also holds for AWGN [9] and Rayleigh fading channels.

Let $A_{v_{1}}$ and $\bar{A}_{v_{1}}$ be the areas under the EXIT function of $f_{v_{1}}(i)$ and $f_{v_{1}}^{-1}(i)$, where $i \in[0,1]$ which can be defined as $A_{v_{1}}=\int_{0}^{1} f_{v_{1}}(i) d i$ and $\bar{A}_{v_{1}}=\int_{0}^{1} f_{v_{1}}^{-1}(i) d i=1-A_{v_{1}}$, respectively. Therefore the area $\bar{A}_{v_{1}}$ under the inverse of the EXIT function is approximately equivalent to the coding rate, $\bar{A}_{v_{1}} \approx R_{o}$. Since the IrURC has a coding rate of unity, the area $A_{v_{2}}$ under the combined inner component block's EXIT curve in Figure 1 can be defined as $A_{v_{2}} \approx C_{\text {channel }}$, where $C_{\text {channel }}$ is the uniform discrete-input channel capacity. Our aim is to create a near-capacity design associated with a narrow EXIT tunnel between the inner and outer EXIT function, reaching the $(1,1)$ convergence point, which typically yields an infinitesimally low BER.

\section{IRREGULAR COMPONENTS}

\section{A. Irregular Outer Component}

We introduce a hybrid combination of irregular repetition codes and convolutional codes for the outer IrCC. First, we employ the 17-component IrCC proposed by Tüchler and Hagenauer [8], which consists of different-rate components created from a mother $\mathrm{CC}$ by puncturing, as indicated by the dotted lines in Figure 2. The original memory-4 IrCC exhibits a near-horizontal portion in the EXIT chart. In order 


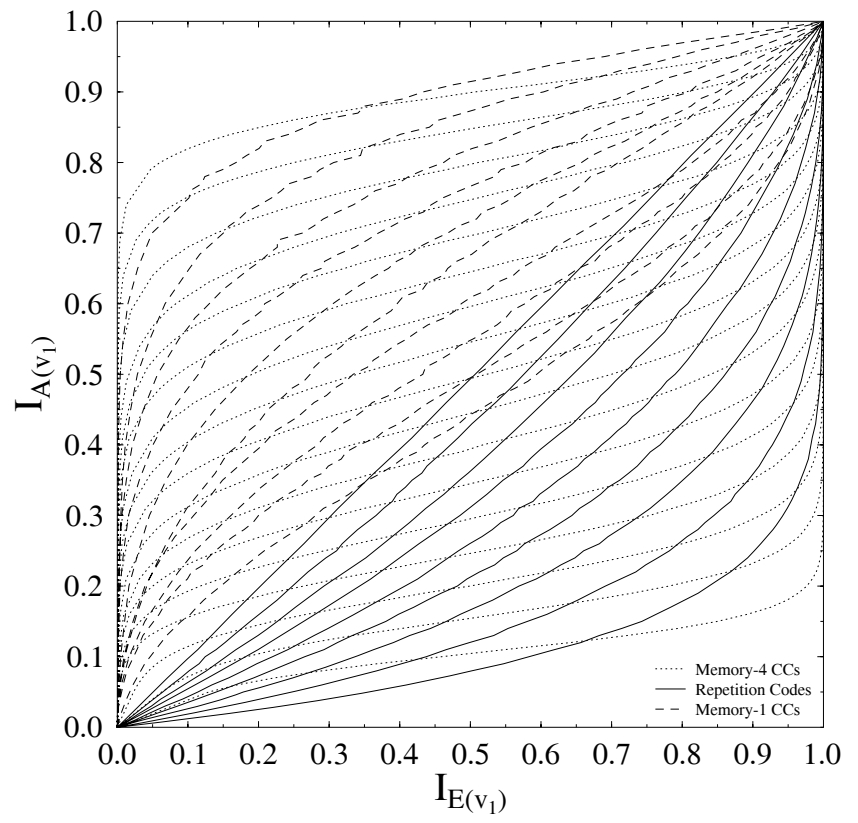

Fig. 2. EXIT functions of the 36 subcodes of the IrCC scheme.

to be able to match the EXIT curves more accurately, we intoduce a range of more diverse-shaped outer EXIT functions, particularly along the diagonal region of the EXIT chart. This can be achieved by invoking weaker codes having a lower memory.

Accordingly, memory-1 CCs are also incorporated in the IrCC scheme, which have a simple two-state trellis diagram. The Generator Polynomials (GPs) of this memory-1 0.5rate mother code are defined as $\left(1, G_{1} / G_{2}\right)$, where we have $G_{1}=D$ and $G_{2}=1$. For lower code rates, an extra output GP, namely $G_{3}$ is used, where we have $G_{3}=G_{2}$. For higher code rates, the puncturing pattern of the memory-4 IrCC is employed [8]. This way we generate 10 additional EXIT functions, as shown by the dashed lines in Figure 2 , spanning the range of $[0.45,0.9]$, with a step size of 0.05 . The 10 memory-1 CCs are specified by the fourtuples $\left\{r,\left(w_{1}, w_{2}, w_{3}\right), l,\left(p_{1}, p_{2}, p_{3}, p_{4}\right)\right\}$, where $r$ represents the code rate, $w_{i}$ specifies how often the GP $G_{i}$ occurs, $l$ is the puncturing period, while the puncturing pattern is defined by $p_{i}$, which is the octal representation associated with $G_{i}$ [8].

A repetition code is a simple memoryless code, which consists of only two codewords, namely the all-zero word and the all-one word. Since it has no memory, the EXIT functions of such repetition codes are diagonally-shaped. In Figure 2, we have nine different-rate repetition codes as indicated by the solid lines and spanning the code-rate range of $[0.1,0.5]$ again, with a step size of 0.05 .

Each of these $P=36$ subcodes encodes a specific fraction of the bit stream $u_{1}$ of Figure 1 according to a specific weighting coefficient $\alpha_{i}, i=1,2, \ldots, 36$. More specifically, let us assume that there are $L$ number of encoded bits $v_{1}$ in Figure 1 , where each subcode $i$ encodes a specific input fraction of $\alpha_{i} r_{i} L$ and generates $\alpha_{i} L$ encoded bits using a coding rate of $r_{i}$. Let us assume that there are $P$ number of subcodes and that the following conditions must be satisfied:

$$
\sum_{i=1}^{P} \alpha_{i}=1,
$$

where $R_{o}=\sum_{i=1}^{P} \alpha_{i} r_{i}$ with $\alpha_{i} \in[0,1], \quad \forall i$ and $R_{o}$ is the average outer code rate. The EXIT functions of all $P=36$ IrCC subcodes are shown in Figure 2.

\section{B. Irregular Inner Component}

In order to generate a narrow but nonetheless open EXIT chart tunnel, which leads to the point of convergence at $\left(I_{A}, I_{E}\right)=(1,1)$, we have to design inner EXIT functions, which match the shape of those in Figure 2 and exhibit a wide variety of EXIT characteristic shapes.

The IrURC scheme consists of three URCs, each having a different memory length. We evaluated the EXIT chart of all possible combinations of up to three different-memory URCs and then selected the three most dissimilar URC EXIT functions, having a generator polynomial $\left(G_{1}, G_{2}\right)$ of $(2,3)_{8}$, $(4,7)_{8}$ and $(16,17)_{8}$. We term them as $\mathrm{URC}_{1} \mathrm{URC}_{2} \mathrm{URC}_{3}$, respectively.

Finally, the IrMapper of Figure 1 consists of irregular mapping schemes, each invoking a different bit-to-symbol mapping strategy. Here, we consider an 8PSK constellation and employ four different mapping schemes, which exhibit dissimilar EXIT functions, namely Gray Mapping (GM), Ungerböck's Partitioning (UP), Block Partitioning (BP) and Mixed Partitioning (MP) [11].

We define the combination of 12 inner components as $\mathrm{UM}_{i}$, where $i \in\{1,2, \ldots, 12\}$ in which the combination constitutes a set of $\left\{\mathrm{UM}_{1}, \mathrm{UM}_{2}, \ldots, \mathrm{UM}_{12}\right\}=\left\{\mathrm{URC}_{1} / \mathrm{GM}, \mathrm{URC}_{2} / \mathrm{GM}\right.$, $\mathrm{URC}_{3} / \mathrm{GM}, \quad \mathrm{URC}_{1} / \mathrm{UP}, \quad \mathrm{URC}_{2} / \mathrm{UP}, \quad \mathrm{URC}_{3} / \mathrm{UP}, \quad \mathrm{URC}_{1} / \mathrm{BP}$, $\left.\mathrm{URC}_{2} / \mathrm{BP}, \mathrm{URC}_{3} / \mathrm{BP}, \mathrm{URC}_{1} / \mathrm{BP}, \mathrm{URC}_{2} / \mathrm{BP}, \mathrm{URC}_{3} / \mathrm{BP}\right\}$. With the IrURC and IrMapper schemes defined, we proceed by creating $Q=12$ different EXIT functions for the inner decoder components, each invoking a different combination of the IrURC and IrMapper schemes.

The EXIT functions of the $Q=12$ combined inner IrURCIrMapper components are plotted in Figure 3 for $E_{b} / N_{0}=$ $5.3 \mathrm{~dB}$. The weighting coefficients are defined as $\beta$, satisfying the following conditions:

$$
\sum_{i=1}^{Q} \beta_{i}=1 \quad \text { and } \beta_{i} \in[0,1], \quad \forall i .
$$

\section{EXIT CHART MATCHING}

We adopt the EXIT chart matching algorithm of [8] to jointly match the EXIT functions of the irregular inner and outer components to each other, as detailed in Section IV-B. The EXIT functions to be considered are described in Section III. More explicitly, we intend to minimise the square of the error function of $e(i)=\left[f_{v_{2}}\left(i, E_{b} / N_{0}\right)-f_{v_{1}}^{-1}(i)\right]$.

Furthermore, the matching error has to be larger than zero and may be expressed as [8] $J\left(\alpha_{1}, \ldots, \alpha_{P}\right)=\int_{0}^{1} e(i)^{2} d i$ or $J\left(\beta_{1}, \ldots, \beta_{Q}\right)=\int_{0}^{1} e(i)^{2} d i$ where $e(i)>0, \forall i \in[0,1]$, and 


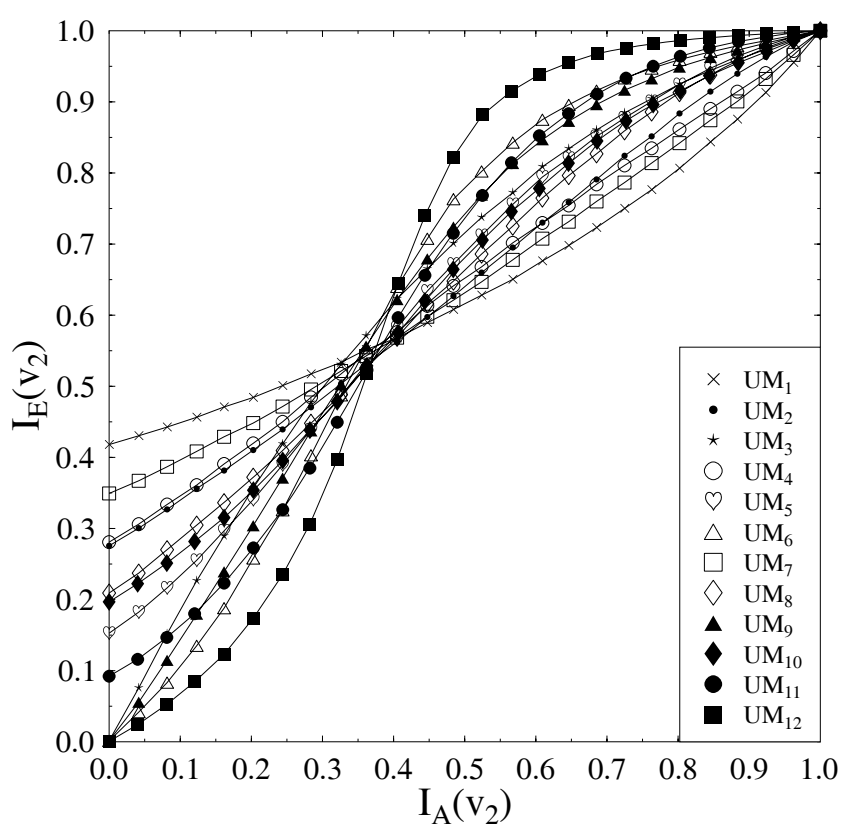

Fig. 3. EXIT functions of the 12 subcodes of the IrURC-IrMapper scheme.

$Q$ or $P$ is the number of irregular subcodes used by either the inner or the outer components, respectively, depending on where the matching process is executed. We term this constraint as condition $C_{1}$. Another constraint we impose here is that of ensuring that Equations (1) and (2) are fulfilled and we term this as condition $C_{2}$ and $C_{3}$, respectively.

The joint EXIT chart matching algorithm designed for both the inner and outer components is adapted from [8] in order to find the optimal value of $\alpha_{\text {opt }}$ and $\beta_{\text {opt }}$, which can be summarised as follows:

Step 1: Create the $P$ outer components of the $\operatorname{IrCC}$ and the $Q$ inner UMs.

Step 2: Select one out of $Q \mathrm{UMs}$, as the inner component to be used.

Step 3: Select an initial outer coding rate $R_{0}$, to be employed in the EXIT-chart matching algorithm of [8]. This $R_{0}$ is the initial $R_{I R}$ to be placed in the matrix $\mathbf{d}$ as detailed in [8].

Step 4: Employ the EXIT chart matching algorithm [8], subject to the constraints of $C_{1}$ and $C_{2}$.

Step 5: Repeat Step 3 and Step 4 iteratively, until a sufficiently high initial rate $R_{0}$ is obtained.

Step 6: Record the resultant outer EXIT curve.

Step 7: Invoke the EXIT chart matching algorithm for finding the best weights of the $Q$ number of UMs to match the the IrCC's outer EXIT curve of Step 6, in order to obtain $\beta_{\text {opt }}$. This process is subject to the constraints of $C_{1}$ and $C_{3}$.

Step 8: Record the resultant inner EXIT curve and repeat the EXIT chart matching process of Step 4, Step 6 and Step 7, each time with a small increment of $R_{0}$, until no more increment is possible.

Step 9: Activate the EXIT matching algorithm to find the best-matching outer code EXIT chart function for the target inner code EXIT function, this time using

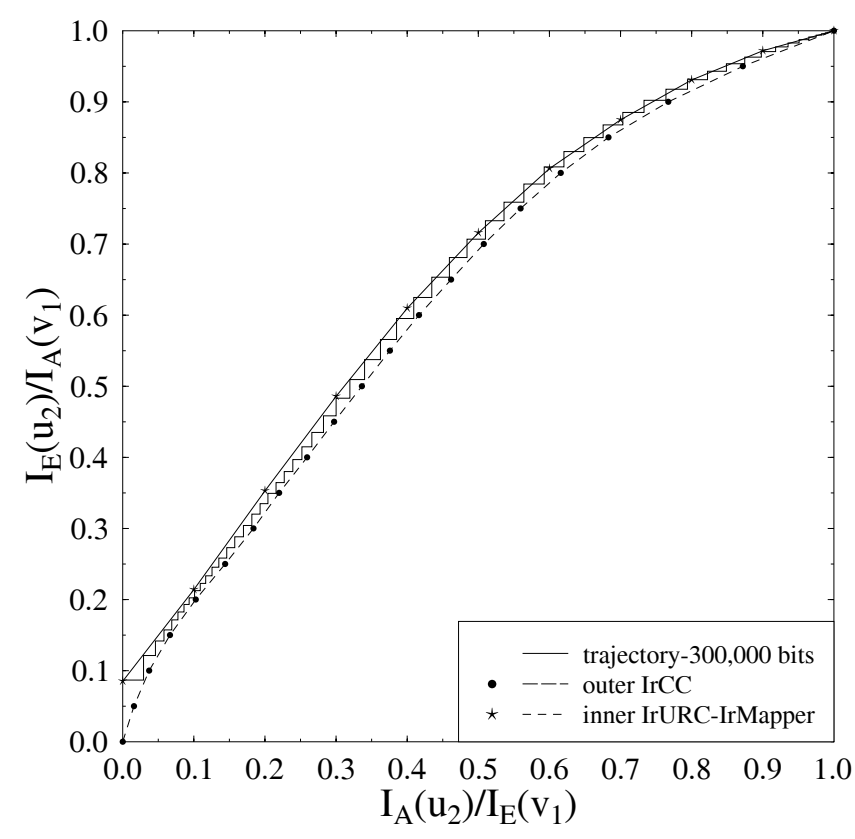

Fig. 4. EXIT functions and the trajectory of the Ir-BICM-ID schemes designed for transmission over an uncorrelated Rayleigh fading channel at $E_{b} / N_{0}=5.3 \mathrm{~dB}$. The overall code rate is 0.635 , the effective throughput is $1.905 \mathrm{bit} / \mathrm{symbol}$ and the modulation scheme is 8PSK.

one out of $Q$ UMs, which was not used in Step 2. Repeat Step 4, Step 6, Step 7 and Step 8, until all the $Q$ number of UMs were tested.

Step 10: Terminate the algorithm, choose the best values of $\alpha_{\text {opt }}$ and $\beta_{\text {opt }}$, yielding the highest possible outer coding rate.

\section{Simulation Results}

In this section we embark on characterising the proposed Ir-BICM-ID scheme in terms of its EXIT chart convergence behaviour for transmission over the uncorrelated Rayleigh fading channel. Let us employ the EXIT matching algorithm described in Section V, invoking the IrCC, IrURC as well as IrMapper schemes. Since the inner IrURC component has a unity rate by definition, the effective throughput of the IrBICM-ID scheme may be expressed as $\eta=R \cdot \log _{2} B$ bits per channel use, where $B$ is the number of constellation points and $R$ denotes the coding rate of the outer IrCC. The relationship of the $\operatorname{SNR}\left(E_{c} / N_{0}\right)$ and $E_{b} / N_{0}$ can be represented as $E_{c} / N_{0}=E_{b} / N_{0} \cdot \eta$, where $N_{0}$ is the noise power spectral density and $E_{c}$ as well as $E_{b}$ denotes the transmit energy per channel symbol use and per bit of source information, respectively.

We observe from Figure 4 that the open EXIT tunnel of the resultant scheme is narrow but still open and hence reaches the point of convergence at $\left(I_{A}, I_{E}\right)=(1,1)$. However, since the number of iterations required is increased, the decoding complexity is also increased. Figure 4 illustrates furthermore that the decoding trajectory matches the inner and outer EXIT functions and evolves within the narrow tunnel, reaching the $\left(I_{A}, I_{E}\right)=(1,1)$ point of convergence for an interleaver length of 300,000 . 


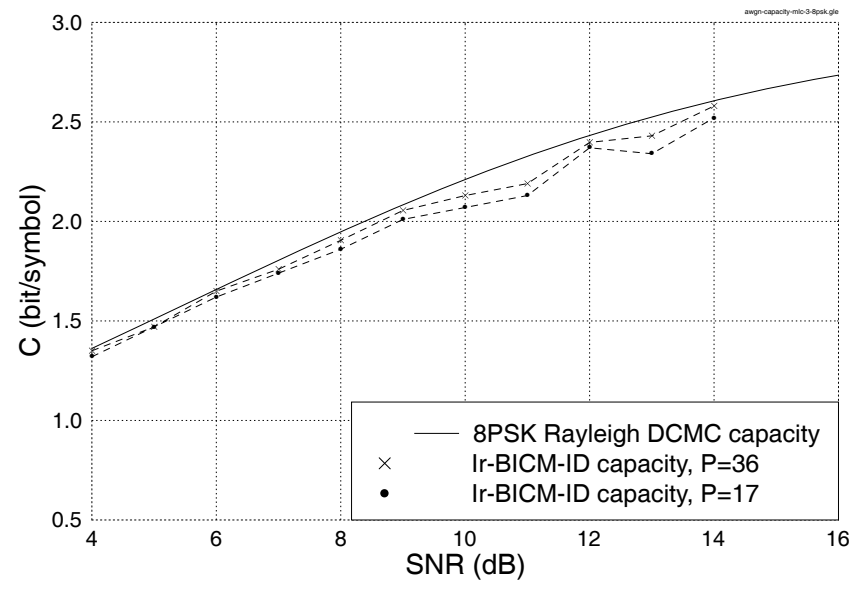

Fig. 5. The maximum effective throughput of the proposed Ir-BICM-ID scheme in comparison to the theoretical DCMC capacity plot. The transmission is over the 8PSK-modulated uncorrelated Rayleigh fading channel.

Note that the proposed Ir-BICM-ID scheme has advantages over the bit-interleaved irregular modulation scheme of [6], when we further explore the effects of various mapping schemes combined with URCs having different memory lengths. This gives us the flexibility of adjusting the EXIT curve shape in order to achieve a low BER, without having to change the number of modulated constellation points, which would require complex, state-of-the-art AMC. Furthermore, we employ the joint EXIT curve matching algorithm of Section $\mathrm{V}$ to produce flexible inner and outer component codes. The complexity imposed by the iterations between the IrURC and IrMapper schemes is low compared to that of the outer IrCC.

The EXIT function of Figure 4 was recorded for the EXIT chart matching algorithm of Section $\mathrm{V}$ for an overall coding rate of 0.635 . The resultant weighting coefficients of $\alpha_{\text {opt }}$ and $\beta_{\text {opt }}$ are $\alpha_{\text {opt }}=\left[\alpha_{1}, \ldots, \alpha_{36}\right]=\left[\alpha_{3}^{0.0311597}, \alpha_{12}^{0.0293419}\right.$, $\left.\alpha_{13}^{0.0228237}, \alpha_{15}^{0.0309682}, \alpha_{23}^{0.0837582}, \alpha_{27}^{0.404757}, \alpha_{36}^{0.404757}\right]$ and $\beta_{\text {opt }}=\left[\beta_{1}, \ldots, \beta_{12}\right]=\left[\beta_{3}^{0.340268}, \beta_{5}^{0.550512}, \beta_{9}^{0.109219}\right]$, where $\alpha_{i}^{j}$ and $\beta_{i}^{j}$ refer to the weighting coefficient of $\alpha_{i}$ and $\beta_{i}$ having a value of $j$. Those components having a weighting coefficient of zero are omitted in the expression for clarity.

The theoretical Discrete-input Continuous-output Memoryless Channel's (DCMC) capacity [12] is plotted in comparison to the maximum achievable capacity of the proposed Ir-BICMID scheme in Figure 5. Since the EXIT functions of the 17 subcodes [8] in Figure 2 consist of memory-4 CCs, the EXIT curves exhibit a rather gently sloping, near-horizontal shapes in their middle section, as seen in Figure 2. Therefore the matching process exhibits a higher discrepancy from the DCMC's capacity. When introducing the whole range of $P=36$ subcodes for the new hybrid IrCCs, we attain a closer match to the channel capacity, as shown by the cross-markers of Figure 5. Note that the achieveable rate of the Ir-BICM-ID scheme is close to the DCMC's capacity. For example, at SNR $=6 \mathrm{~dB}$ the discrepancy between the theoretical capacity and the proposed coded modulation scheme's curve is less than $0.1 \mathrm{~dB}$ in Figure 5.

Figure 6 shows the BER performance of the Ir-BICMID scheme for different interleaver lengths. The DCMC's

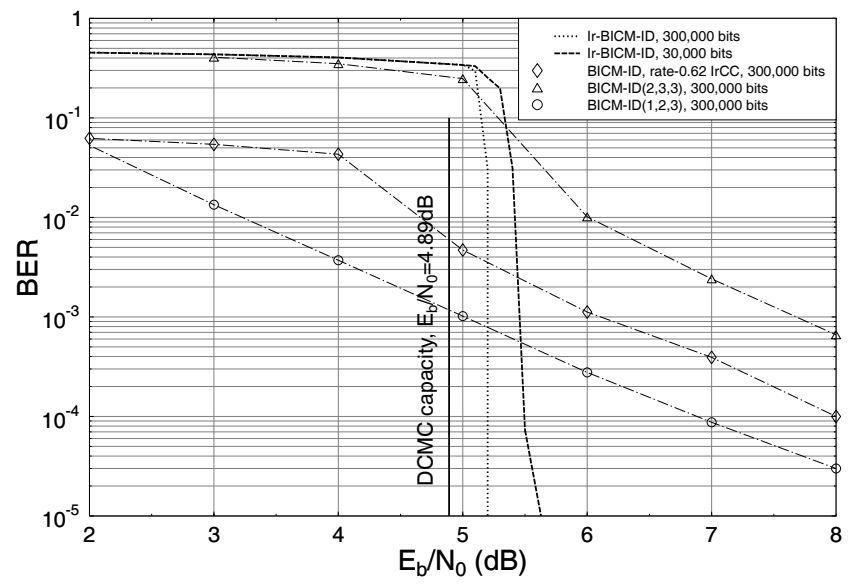

Fig. 6. The BER performance of rate-0.635 Ir-BICM-ID schemes at an effective throughput of $1.905 \mathrm{bit} / \mathrm{symbol}$, using various interleaver lengths and that of the classic 8PSK UP BICM-ID [1], employing a coding rate of $R=1 / 2$ and $2 / 3$, respectively. A BER benchmarker of BICM-ID scheme invoking rate-0.62 outer IrCC proposed in [8] employing UP mapper is also attained.

capacity limit is $E_{b} / N_{0}=4.89 \mathrm{~dB}$ at a throughput of 1.905 bit/symbol using 8PSK. By employing a 300,000 bit interleaver, the Ir-BICM-ID design becomes capable of achieving an infinitesimally low BER about $0.32 \mathrm{~dB}$ away from the theoretical capacity limit, as shown in Figure 5. When we decrease the interleaver length by a factor of 10 to 30,000 bits, the performance degrades, but still remains within about $0.75 \mathrm{~dB}$ of the capacity. We include the classic $1 / 2$ - and $2 / 3$ rate BICM-ID benchmarker scheme [1] having a 300,000-bit interleaver length as well as the BICM-ID scheme employing the $P=17$ IrCC [8] of rate- 0.62 . All the benchmarkers exhibit BER $>10^{-5}$ even at $E_{b} / N_{0}=8 \mathrm{~dB}$, which is relatively far from the capacity limit of $E_{b} / N_{0}=4.89 \mathrm{~dB}$.

The complexity of the scheme can be quantified in terms of the total number of trellis states of both the IrCC and IrURC schemes. A total of about $6 \times 10^{8}$ IrURC and IrCC decoder trellis-states are required for an infinitesimally low BER at an interleaver length of 300,000 bits and $E_{b} / N_{0}=5.3 \mathrm{~dB}$, i.e. for performing close to capacity. However, at high $E_{b} / N_{0}$, such as $E_{b} / N_{0}=6.5 \mathrm{~dB}$ for example, the number of trellis states involved substantially decreased, to around $1.8 \times 10^{8}$. The irregular structure of the schemes may not necessarily impose a higher complexity, since the same MAP algorithm is applied for their decoding as for regular codes. However, a potentially high complexity is imposed due to the extra iterations imposed by their narrow EXIT tunnel.

\section{CONCLUSiOnS}

In conclusion, a novel Ir-BICM-ID scheme was proposed. This scheme invokes the combined IrCC, IrURC and IrMapper arrangement of Figure 1. Diverse-shaped EXIT curves were produced for both the inner and outer component codes. A useful joint inner-outer EXIT matching algorithm was employed for creating a narrow, but still open EXIT tunnel, which indicated near-capacity operation. Our results demonstrate that in contrast to the classic BICM-ID schemes, the proposed arrangement is capable of attaining an infinitesimally low BER. The BER performance seen in Figure 6 indicates 
reliable operation within about $0.32 \mathrm{~dB}$ of the capacity at $E_{b} / N_{0}=4.89 \mathrm{~dB}$, using the weighting coefficients of $\alpha_{\mathbf{o p t}}$ and $\beta_{\text {opt }}$ obtained in Section VI for an interleaver length of 300,000 .

\section{ACKNOWLEDGEMENT}

The financial support of the European Union (EU) in the framework of the Optimix project and that of the EPSRC, UK is gratefully acknowledged.

\section{REFERENCES}

[1] X. Li and J. A. Ritcey, "Bit-interleaved coded modulation with iterative decoding," IEEE Commun. Lett., vol. 1, no. 6, pp. 169-171, Nov. 1997.

[2] L. Hanzo, T. H. Liew, and B. L. Yeap, Turbo Coding, Turbo Equalisation and Space-Time Coding. Chichester, UK; Piscataway, NJ, USA: John Wiley, IEEE Press, 2002.

[3] L. Hanzo, S. X. Ng, T. Keller, and W. Webb, Quadrature Amplitude Modulation: From Basics to Adaptive Trellis-Coded, Turbo Equalised and Space-Time Coded OFDM, CDMA and MC-CDMA Systems, 2nd ed. Chichester, England: John Wiley and Sons Ltd. and IEEE Press, 2004.

[4] S. T. Brink, "Convergence behavior of iteratively decoded parallel concatenated codes," IEEE Trans. Commun., vol. 49, no. 10, pp. 1727-1737, Oct. 2001.
[5] N. H. Tran and H. H. Nguyen, "Signal mappings of 8-ary constellations for BICM-ID systems over a Rayleigh fading channel," IEICE Trans. Lett. Commun., vol. E88-B, no. 10, pp. 4083-4086, Oct. 2005.

[6] F. Schreckenbach and G. Bauch, "Bit-interleaved coded irregular modulation," European Trans. Telecommun., vol. 7, no. 2, pp. 269-282, Apr. 2006.

[7] M. Tüchler, "Design of serially concatenated systems depending on the block length," IEEE Trans. Commun., vol. 52, no. 2, pp. 209-218, Feb. 2004.

[8] M. Tüchler and J. Hagenauer, "EXIT charts of irregular codes," in Proc. IEEE Conf. Inform. Sciences Syst., pp. 748-753, Mar. 2002.

[9] M. Tüchler, "Convergence prediction for iterative decoding of threefold concatenated systems," in Proc. IEEE Global Telecommun. Conf., vol. 2, pp. 1358-1362, Nov. 2002.

[10] A. Ashikhmin, G. Kramer, and S. T. Brink, "Extrinsic information transfer functions: model and erasure channel properties," IEEE Trans. Inform. Theory, vol. 50, no. 11, pp. 2657-2673, Nov. 2004.

[11] U. Wachsmann, R. F. H. Fischer, and J. B. Huber, "Multilevel codes: theoretical concepts and practical design rules," IEEE Trans. Inform. Theory, vol. 45, pp. 1361-1391, July 1999.

[12] S. X. Ng and L. Hanzo, "On the MIMO channel capacity of multidimensional signal sets," IEEE Trans. Veh. Technol., vol. 55, no. 2, pp. 528-536, Mar. 2006. 